\title{
INFLUENCE OF THE STELLAR WINDS ON THE EVOLUTION
}

\section{OF THE PLANETARY NEBULA NUCLEI.}

\author{
S.K.GÓRNY
}

Nicolaus Copernicus Astronomical Center, Chopina 12/18, 87-100 Toruń, POLAND

A grid of homogeneous models of evolution of hydrogen burning planetary nebulae nuclei, assuming different stellar winds and the zero points for the post-AGB evolution, have been constructed from original Schönberners tracks. Following a simplified line-driven wind theory the mass loss rate has been adopted to be

$$
\dot{M}=\alpha \frac{L}{c \cdot V_{\infty}}
$$

with $\alpha=0.50,0.25,0.12,0.06$ and $V_{\infty}$ changing from $V_{\infty}=1 \cdot V_{e s c}$ at $T_{\text {eff }}=10^{3.6} \mathrm{~K}$ to $V_{\infty}=4 \cdot V_{e s c}$ at $T_{e f f}=10^{5.1} \mathrm{~K}$. The zero points for evolution ("superwind" cessation) have been chosen at the moments when the fundamental mode period of pulsations is $P_{0}=100,75,50,25$ days.

Using a simple model of a coupled evolution of a planetary nebula and its central star the observational characteristics and the various diagrams usually used for investigation of the planetaries have been calculated (e.g. $M_{V}-t_{e x p}$-see figures).
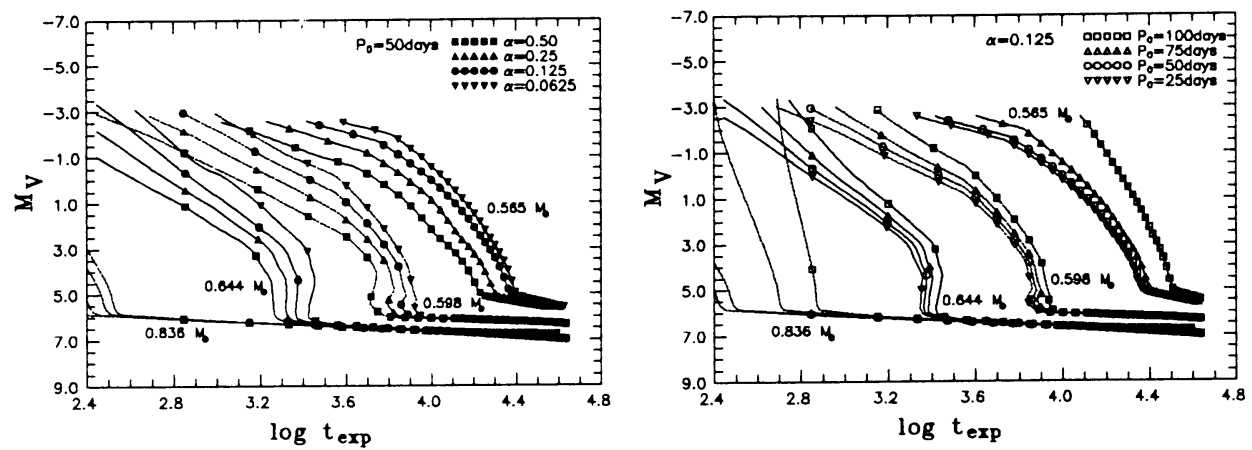

Figure:The influence of stellar winds (left) and starting points (right) on the PNN evolution.

From diagrams it can be seen that modifications connected with different stellar winds are generally more significant than the moment of "superwind" cessation. The zero point is important only if the "superwind" ends at low temperatures but even for this case a high mass loss may significantly speed up the evolution.

It is worth to notice that although the mass loss and the zero points have substantially been modified the new time scale of evolution for each nuclei of a given mass is generally in agreement with already existing models.

The sensitivity of the tracks positions to the adopted wind scenario has been investigated on the observational diagrams constructed applying the standard Zanstra and Shklovski methods for deriving stellar parameters. An extensive discussion of the problem will be published in near future. 